\title{
Noncirrhotic hepatocellular carcinoma: derivation from hepatocellular adenoma? Clinicopathologic analysis
}

\author{
Ta-Chiang Liu ${ }^{1}$, Neeta Vachharajani ${ }^{2}$, William C Chapman ${ }^{2}$ and Elizabeth M Brunt ${ }^{1}$ \\ ${ }^{1}$ Department of Pathology and Immunology, Washington University School of Medicine, Saint Louis, MO, \\ USA and ${ }^{2}$ Department of Surgery, Washington University School of Medicine, Saint Louis, MO, USA
}

\begin{abstract}
The majority of hepatocellular carcinomas arise in background chronic liver disease, particularly cirrhosis. The pathogenesis of noncirrhotic hepatocellular carcinomas remains unclear. While malignant transformation reportedly occurs in $<15 \%$ of hepatocellular adenoma, the prevalence of noncirrhotic hepatocellular carcinomas arising from a pre-existing adenoma is a challenge to study. Cirrhotic hepatocellular carcinoma and hepatocellular adenoma may be subclassified by molecular pathways, but little is known in noncirrhotic hepatocellular carcinoma. We aim to delineate clinical, morphologic and immunohistochemical features of noncirrhotic hepatocellular carcinoma to evaluate for possible derivation from hepatocellular adenoma. We evaluated the clinicopathologic features of 74 noncirrhotic hepatocellular carcinomas from 72 patients for underlying clinical conditions and immunohistochemical markers known to be associated with hepatocellular adenoma. Men were more commonly affected (59\%); however, in the $<50$-year-old group, women predominated (8:1). The age range was wide: 18-83 years; median-64 years. Underlying liver diseases were identified in only $7 \%$; however, $25 \%$ had diabetes mellitus, $69 \%$ were overweight or obese and $58 \%$ had metabolic syndrome. Only $50 \%$ of the noncirrhotic hepatocellular carcinoma were encapsulated. As published in hepatocellular adenoma, multifocality and larger tumor size were more common in liver fatty acid-binding protein-negative noncirrhotic hepatocellular carcinoma. Beta-catenin nuclear positivity was uncommon (5\%), and was restricted to hepatocellular carcinomas in older men. Serum amyloid A positivity was not restricted to any subtype. In summary, we present the largest series to date examining noncirrhotic hepatocellular carcinoma. We evaluated these with current hepatocellular adenoma subclassification markers for possible associations. Thirty percent of the 74 noncirrhotic hepatocellular carcinoma had some clinical, morphological or immunophenotypical associations currently described in hepatocellular adenoma. Our data also confirm the association of noncirrhotic hepatocellular carcinoma in middle-aged to elderly men, an association with metabolic syndrome, and, as with hepatocellular adenoma, that women predominated in the noncirrhotic hepatocellular carcinoma subjects $<\mathbf{5 0}$ years of age.
\end{abstract}

Modern Pathology (2014) 27, 420-432; doi:10.1038/modpathol.2013.148; published online 20 September 2013

Keywords: $\beta$-catenin; cirrhosis; glutamine synthetase; liver fatty acid-binding protein; metabolic syndrome; serum amyloid $A$

The worldwide incidence of hepatocellular carcinoma is increasing. ${ }^{1}$ The most common associations with hepatocellular carcinoma are chronic liver disease due to viral hepatitis $\mathrm{B}$ and $\mathrm{C}$ and alcohol; nonalcoholic fatty liver with or without cirrhosis is

Correspondence: Professor EM Brunt, MD, Department of Pathology and Immunology, Washington University School of Medicine, 660 South Euclid Avenue, Campus Box 8118, Apt 1235, St Louis, MO 63110, USA.

E-mail: ebrunt@path.wustl.edu

Received 26 April 2013; revised 1 July 2013; accepted 2 July 2013; published online 20 September 2013 also increasingly recognized in association with hepatocellular carcinoma. $^{2-4}$ Any chronic liver disease that results in cirrhosis however potentially shares common final genetic pathways to hepatocellular carcinomas through cirrhosis and dysplastic nodules. ${ }^{5}$ Interestingly, the etiopathogenesis of noncirrhotic hepatocellular carcinoma remains unknown. ${ }^{3,6,7}$

In addition, noncirrhotic hepatocellular carcinoma presents several challenges to diagnosis and management. There are no established guidelines for the detection of hepatocellular carcinoma in patients that are not cirrhotic, as there are for patients with cirr- 
hosis; ${ }^{8,9}$ thus, affected patients are often diagnosed because of symptoms rather than in screening and they commonly have larger, higher-stage tumors at the time of presentation than do patients with cirrhosis. Histopathological difficulties may arise with distinctions between well-differentiated hepatocellular carcinoma and hepatocellular adenoma, particularly in small biopsy specimens, as the latter, by definition, arise in a background of noncirrhotic liver. ${ }^{10}$

Molecular studies have resulted in the following subclassification of hepatocellular adenoma with reproducible genotype-phenotype correlations: ${ }^{11,12}$ (i) adenomas in which the tumor suppressor hepatocyte nuclear factor- $1 \alpha$ is inactivated lack expression of liver fatty acid-binding protein by immunohistochemistry; the tumors are steatotic. These are the most common hepatocellular adenoma to be multiple. ${ }^{13}$ (ii) The least-common hepatocellular adenoma are those with $\beta$-catenin mutation, detected as nuclear and cytoplasmic $\beta$-catenin reactivity. This is accompanied by diffuse, strong glutamine synthetase reactivity by immunohistochemistry; ${ }^{10}$ of note, a recent study has suggested the possibility that this entity may represent a very early hepatocellular carcinoma. ${ }^{14}$ (iii) Hepatocellular adenoma with telangiectatic vessels, inflammatory cell collections and inflammatory protein expression, such as serum amyloid A or C-reactive protein ${ }^{12}$ by immunohistochemistry, comprise the third group. These tumors, formerly termed telangiectatic focal nodular hyperplasia, are clonal $^{15}$ and molecular characterization of aberrant inflammatory pathway activation has been shown. ${ }^{16,17}$ (iv) Other 'unclassified' hepatocellular adenoma comprise the fourth type of hepatocellular adenoma. Among the four groups, the $\beta$-cateninactivated hepatocellular adenoma have a higher association with progression to hepatocellular carcinoma, and are also the hepatocellular adenoma that may be more often found in men. ${ }^{13}$

While the demographics and potential risk factors, as well as immunohistochemical staining patterns for the above-mentioned immunohistochemistry markers are well documented in hepatocellular adenoma, little is known of the potential reactivity of these markers in noncirrhotic hepatocellular carcinoma. Study of the staining patterns in noncirrhotic hepatocellular carcinoma will not only aid in differential diagnosis but may provide insight into pathogenesis of noncirrhotic hepatocellular carcinoma. The aim of the current study is therefore to characterize the clinical and pathologic features and immunohistochemistry staining patterns of these markers in a large number of noncirrhotic hepatocellular carcinoma, and to evaluate the possibility of association with pre-existing hepatocellular adenoma based on the clinical information and immunohistochemistry patterns of reactivity. The assumption was made in advance that if hepatocellular adenoma preceded the hepatocellular carcinomas, ie, if the hepatocellular carcinoma had developed from malignant transformation of hepatocellular adenoma, the immunohistochemistry markers of the hepatocellular adenoma could possibly be retained. Clinical outcomes of these noncirrhotic hepatocellular carcinoma patients were compared with recent reports in the literature. ${ }^{18}$

\section{Materials and methods}

\section{Patients}

All resected or transplanted noncirrhotic (Scheuer fibrosis stage 0-3) hepatocellular carcinomas cases between 1990 and 2009 identified by a computer database search of the Department of Pathology and Immunology, Washington University School of Medicine, were identified. Exclusions were serologic detection of hepatitis $\mathrm{B}$ infection and inadequate material for the study. Medical records were reviewed, and pertinent demographic data (age, gender, underlying diseases including features of metabolic syndrome), and follow-up data (recurrencefree survival and overall survival) were extracted. The study was approved by the Institutional Review Board of Washington University School of Medicine.

\section{Histopathology}

Morphologic evaluation was performed on the tumor sections that had been previously submitted for standard-of-care evaluation; these ranged from 1 (small tumor) to $>20$ sections per case. Peritumoral liver tissue was examined on at least 2 blocks (small tumors) to $>6$ blocks for each case. The morphologic studies were performed by the two pathologists. After evaluation of entire cases, 1-2 representative tumor blocks/case were chosen that showed the least amount of necrosis and/or hemorrhage, and that included background liver for immunohistochemistry. Tumor classification was based on 2010 World Health Organization, ${ }^{19}$ and combined hepatocellular carcinoma-cholangiocarcinoma cases were excluded from the study. Fibrosis staging (0-4) was determined using Scheuer criteria. $^{20}$ Tumor grading was Edmondson-Steiner criteria. Steatosis in both the liver and the tumor were individually graded (0: none; 1: $<33 \% ; 2: 33-66 \% ; 3:>66 \%$ ), and in cases where background, nontumoral liver showed grade 2-3 steatosis, nonalcoholic fatty liver disease activity score was determined; ${ }^{21}$ a diagnosis of steatohepatitis was rendered if present. Histopathologic features specifically noted for classification as potential hepatocellular adenoma included the following: uniform bland tumor cytology with little to no nuclear pleomorphism or nucleoli, lack of mitoses, intratumoral large droplet steatosis, prominent telangiectasias/peliosis, intratumoral foci of chronic inflammation associated with fibrovascular cores. Steatosis, peliosis and inflammatory 
foci were not required variables, whereas the bland nature of the tumors and lack of mitoses were.

\section{Immunohistochemistry}

Prior to immunohistochemistry, antigen retrieval was performed using $1 \mathrm{mM}$ EDTA at $\mathrm{pH}$ 8.0. A panel of immunohistochemistry was performed using a Benchmark XT autostainer (Ventana; Tucson, AZ, USA). The antibodies included: glutamine synthetase (1: 200; BD Biosciences; San Jose, CA, USA), liver fatty acid-binding protein (1: 50; Abcam; Cambridge, MA, USA), serum amyloid A ( $4 \mu \mathrm{g} / \mathrm{ml}$; DAKO; Carpinteria, CA, USA), TP53 (prediluted $3 \mathrm{mg} / \mathrm{ml}$; Ventana), keratin 19 (K19; prediluted $0.26 \mu \mathrm{g} / \mathrm{ml}$; Cell Marque; Rocklin, CA, USA), $\beta$ catenin (prediluted $3.06 \mu \mathrm{g} / \mathrm{ml}$; Cell Marque), glypican-3 (prediluted $0.5 \mu \mathrm{g} / \mathrm{ml}$; Cell Marque). The Ultraview universal DAB detection system was used for signal detection (Ventana).

\section{Criteria for Determining Noncirrhotic Hepatocellular Carcinoma with Potential Derivation from Hepatocellular Adenoma}

The following criteria were used to define potential derivation of noncirrhotic hepatocellular carcinoma from hepatocellular adenoma: (1) cytologic and/or architectural evidence of co-existing noncirrhotic hepatocellular carcinoma and hepatocellular adenoma in the same lesion; (2) tumor with steatosis lacking liver fatty acid-binding protein staining; (3) diffuse serum amyloid A expression in noncirrhotic hepatocellular carcinoma that also showed telangiectatic vessels within the tumor; (4) nuclear and/or cytoplasmic $\beta$-catenin with diffuse glutamine synthetase staining in the tumor. Tumors that met at least one of the criteria were classified as possible derivation from hepatocellular adenoma.

\section{Statistical Analysis}

For each parameter, a univariate analysis was performed. Unpaired $t$-test was used for comparison between two separate groups. One-way ANOVA test was used for multi-group comparison. Significance was defined as $P<0.05$.

\section{Results}

A total of 72 patients with 74 noncirrhotic hepatocellular carcinomas met enrollment criteria. Fortyfour patients were men (59\%). The ages ranged from 18 to 83 years; mean and median ages were 64 and 65 years, respectively. Subjects $<50$ years were almost all women (8:1, women:men), whereas men predominated in the higher ages (43: 22; both $P<0.05$ ) (Figure 1). Affected men had greater mean age compared with affected women (65.7 years vs

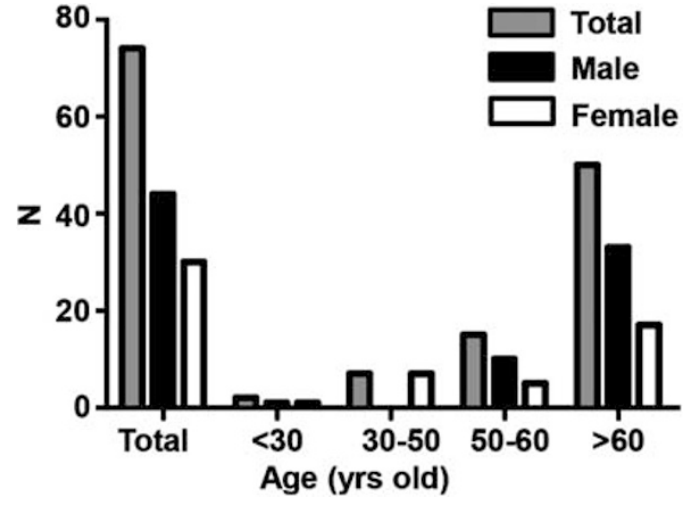

Figure 1 Age and gender distribution of patients with noncirrhotic hepatocellular carcinoma.

59.7 years). Among the 65 patients with available clinical data, diabetes mellitus was documented in $16(25 \%)$. Body mass index $\left(\mathrm{kg} / \mathrm{m}^{2}\right)$ information was available for 55 patients; 19 patients $(35 \%)$ were overweight (body mass index 25-29), and 17 patients $(31 \%)$ were obese (body mass index $\geq 30$ ); thus, only 19 patients (35\%) were within normal limits (body mass index $<25$ ). Applying criteria for metabolic syndrome, ${ }^{22} 15$ of 26 patients with complete clinical data for evaluation had metabolic syndrome (58\%). Five patients (7\%) were diagnosed with hepatitis C. History of more than moderate alcohol use was noted in 6 out of 55 patients (11\%) (Table 1). However, histologic evidence of alcoholic liver disease was documented in only 1 patient (1\%), who had concomitant hepatitis C. Overall, $7 \%$ of patients had underlying viral hepatitis with/ without alcoholic liver disease. Twenty specimens $(27 \%)$ had no fibrosis, $19(27 \%)$ had stage 1 fibrosis (portal expansion), 27 (36\%) had stage 2 fibrosis (periportal fibrosis), and 8 (11\%) had stage 3 fibrosis (bridging fibrosis with nodularity). By definition, none were cirrhotic.

Table 2 summarizes clinicopathologic findings. Fifteen specimens $(20 \%)$ showed varying degrees of steatosis in the nontumor liver; the majority of these livers showed grade 1 steatosis, but $4(5 \%)$ showed grades 2-3 steatosis. Three of 4 corresponding subjects were overweight/obese. No sample was diagnosed as steatohepatitis.

There were 74 hepatocellular carcinomas. The sizes of the resected hepatocellular carcinomas ranged from $1.3 \mathrm{~cm}$ to $24.0 \mathrm{~cm}$, with a mean of $8.1 \mathrm{~cm}$. Fifty-four $(73 \%)$ of the tumors were solitary. Tumor encapsulation was present in only 37 cases $(50 \%)$. Edmondson-Steiner grades were as follows: grade I, 10 (14\%); grade II, 35 (47\%); grade III, 17 $(23 \%)$, and grade IV, $12(16 \%)$. The majority of hepatocellular carcinomas had no lymph-vascular space invasion (49 cases; 66\%). Two cases were fibrolamellar hepatocellular carcinoma (3\%), 1 scirrhous type (1\%), and 5 were inflammatory $(7 \%)$. Varying degrees of steatosis were seen within 7 hepatocellular carcinomas (9\%). Necrosis was 
Table 1 Demography, key histology and immunohistochemistry findings classified by fibrosis stage

\begin{tabular}{|c|c|c|c|c|c|}
\hline Fibrosis staging & 0 & 1 & 2 & 3 & Overall \\
\hline Total $N$ & 20 & 19 & 27 & 8 & 74 \\
\hline Male:female & $10: 10$ & $11: 8$ & $16: 11$ & $7: 1$ & $44: 30$ \\
\hline Age (mean) & 63 & 64.6 & 61.6 & 70.3 & 63.7 \\
\hline Age (range) & $38-82$ & $30-80$ & $18-80$ & $50-83$ & $18-83$ \\
\hline Hepatitis C & 0 & 1 & 3 & 1 & 5 \\
\hline Metabolic Syndrome & $3 / 6(50.0 \%)$ & $6 / 10(60.0 \%)$ & $5 / 8(62.5 \%)$ & $1 / 2(50.0 \%)$ & $15 / 26(57.7 \%)$ \\
\hline Hypertension & $9 / 14(64.3 \%)$ & $13 / 16(81.3 \%)$ & $19 / 21(90.5 \%)$ & $2 / 7(28.6 \%)$ & $46 / 58(74.1 \%)$ \\
\hline Diabetes mellitus & $3 / 16(18.8 \%)$ & $5 / 16(31.3 \%)$ & $6 / 27(22.2 \%)$ & $2 / 6(33.3 \%)$ & $16 / 65(24.6 \%)$ \\
\hline Body mass index (average) & 27.02 & 27.31 & 27.33 & 25.18 & 27.11 \\
\hline$<25$ & $6 / 14(42.9 \%)$ & $4 / 14(28.6 \%)$ & $7 / 21(33.3 \%)$ & $2 / 6(33.3 \%)$ & $19 / 55(34.5 \%)$ \\
\hline $25-29$ & $3 / 14(21.4 \%)$ & $5 / 14(35.7 \%)$ & $8 / 21(38.1 \%)$ & $3 / 6(50.0 \%)$ & $19 / 55(34.5 \%)$ \\
\hline$\geqslant 30$ & $5 / 14(35.7 \%)$ & $5 / 14(35.7 \%)$ & $6 / 21(28.6 \%)$ & $1 / 6(14.3 \%)$ & $17 / 55(30.9 \%)$ \\
\hline Alcohol & $0 / 15(0 \%)$ & $1 / 11(9.1 \%)$ & $5 / 23(21.7 \%)$ & $0 / 6(0 \%)$ & $6 / 55(10.9 \%)$ \\
\hline Tumor size (average)* & 10.0 & 8.6 & 6.7 & 7.08 & 8.10 \\
\hline Tumor size (range) & 1.7-18.0 & 1.6-24.0 & 1.3-19.0 & 3.5-10.5 & $1.3-24.0$ \\
\hline Multifocality & $4 / 20(20.0 \%)$ & $5 / 19(26.3 \%)$ & $8 / 27(29.6 \%)$ & $3 / 8(37.5 \%)$ & $20 / 74(27.0 \%)$ \\
\hline Fibrolamellar subtype & 1 & 0 & 1 & 0 & 2 \\
\hline Inflammatory subtype & 0 & 0 & 5 & 0 & 5 \\
\hline Schirrhous subtype & 0 & 0 & 1 & 0 & 1 \\
\hline \multicolumn{6}{|l|}{ Grade } \\
\hline I & $3 / 20(15.0 \%)$ & 2/19 (10.5\%) & $5 / 27(18.5 \%)$ & 0 & $10(13.5 \%)$ \\
\hline II & $10 / 20(50.0 \%)$ & $11 / 19(57.9 \%)$ & $11 / 27(40.7 \%)$ & $3 / 8(37.5 \%)$ & $35(47.3 \%)$ \\
\hline III & $2 / 20(10.0 \%)$ & $5 / 19(26.3 \%)$ & $6 / 27(22.2 \%)$ & $4 / 8(50.0 \%)$ & $17(23.0 \%)$ \\
\hline IV & $5 / 20(25.0 \%)$ & $1 / 19(5.3 \%)$ & $5 / 27(18.5 \%)$ & $1 / 8(12.5 \%)$ & $12(16.2 \%)$ \\
\hline $\begin{array}{l}\text { Significant steatosis in } \\
\text { tumor* }\end{array}$ & 0 & 0 & $6 / 27(22.2 \%)$ & $1 / 8(12.5 \%)$ & $7(9.5 \%)$ \\
\hline Significant steatosis in liver & $3 / 20(15.0 \%)$ & 0 & $1 / 27(3.7 \%)$ & 0 & $4(5.4 \%)$ \\
\hline \multicolumn{6}{|l|}{ P53 } \\
\hline$<1 \%$ & $15 / 20(75.0 \%)$ & $16 / 19(84.2 \%)$ & $17 / 27(63.0 \%)$ & $4 / 8(50.0 \%)$ & $52(70.3 \%)$ \\
\hline$<10 \%$ & $3 / 20(15.0 \%)$ & $1 / 19(5.3 \%)$ & $5 / 27(18.5 \%)$ & $3 / 8(37.5 \%)$ & $12(16.2 \%)$ \\
\hline$\geqslant 10 \%$ & $2 / 20(10.0 \%)$ & $2 / 19(10.5 \%)$ & $5 / 27(18.5 \%)$ & $1 / 8(12.5 \%)$ & $10(13.5 \%)$ \\
\hline \multicolumn{6}{|l|}{ K19 } \\
\hline$<5 \%$ & $17 / 20(85.0 \%)$ & 18/19 (94.7\%) & $23 / 27(85.2 \%)$ & $7 / 8(87.5 \%)$ & $65(87.8 \%)$ \\
\hline$\geqslant 5 \%$ & $3 / 20(15.0 \%)$ & $1 / 19(5.3 \%)$ & $4 / 27(14.8 \%)$ & $1 / 8(12.5 \%)$ & $9(12.2 \%)$ \\
\hline Beta-catenin & $\begin{array}{c}\text { Patchy: } 1 / 20 \\
(5.0 \%)\end{array}$ & Patchy: 1/19 (5.3\%) & $1 / 27(3.7 \%)$ & Patchy 1/8 (12.5\%) & $4(5.4 \%)$ \\
\hline \multicolumn{6}{|l|}{ Glypican-3 } \\
\hline Negative & $8 / 20(40.0 \%)$ & $7 / 19(36.8 \%)$ & $7 / 27(25.9 \%)$ & 0 & $22(29.7 \%)$ \\
\hline Heterogeneous & 0 & $1 / 19(5.3 \%)$ & 0 & $1 / 8(12.5 \%)$ & $2(2.7 \%)$ \\
\hline Patchy & $8 / 20(40.0 \%)$ & $5 / 19(26.3 \%)$ & $5 / 27(18.5 \%)$ & $4 / 8(50.0 \%)$ & $22(29.7 \%)$ \\
\hline Diffuse & $4 / 22(20.0 \%)$ & $6 / 19(31.6 \%)$ & $15 / 27(55.6 \%)$ & $3 / 8(37.5 \%)$ & $28(37.8 \%)$ \\
\hline \multicolumn{6}{|c|}{ Liver fatty acid binding protein } \\
\hline$<1 \%$ & $5 / 20(25.0 \%)$ & 4/19 (21.1\%) & $8 / 27(29.6 \%)$ & $1 / 8(12.5 \%)$ & $18(24.3 \%)$ \\
\hline$<80 \%$ (patchy/focal) & $5 / 20(25.0 \%)$ & $8 / 19(42.1 \%)$ & $6 / 27(22.2 \%)$ & $5 / 8(62.5 \%)$ & $24(32.4 \%)$ \\
\hline$\geqslant 80 \%$ (diffuse) & $10 / 20(50.0 \%)$ & $7 / 19(36.8 \%)$ & $13 / 27(48.1 \%)$ & $2 / 8(25.0 \%)$ & $32(43.2 \%)$ \\
\hline \multicolumn{6}{|l|}{ Glutamine Synthetase } \\
\hline Negative & 0 & 0 & $1 / 27(3.7 \%)$ & 0 & $1(1.4 \%)$ \\
\hline Heterogeneous & $2 / 20(10.0 \%)$ & $5 / 19(26.3 \%)$ & $8 / 27(29.6 \%)$ & $3 / 8(37.5 \%)$ & $18(24.3 \%)$ \\
\hline Patchy* & $5 / 20(25.0 \%)$ & 0 & 0 & 0 & $5(6.8 \%)$ \\
\hline Diffuse & $13 / 20(65.0 \%)$ & 14/19 (73.4\%) & $18 / 27(66.7 \%)$ & $5 / 8(62.5 \%)$ & $50(67.6 \%)$ \\
\hline \multicolumn{6}{|l|}{ Serum amyloid $A$} \\
\hline $0 \%$ & $10 / 20(50.0 \%)$ & $9 / 19(47.4 \%)$ & $7 / 27(25.9 \%)$ & $4 / 8(50.0 \%)$ & $30(40.5 \%)$ \\
\hline <80\% (patchy/focal) & $6 / 20(30.0 \%)$ & $5 / 19(26.3 \%)$ & $16 / 27(59.3 \%)$ & $3 / 8(37.5 \%)$ & $30(40.5 \%)$ \\
\hline$\geqslant 80 \%$ (diffuse) & $4 / 20(20.0 \%)$ & $5 / 19(26.3 \%)$ & $4 / 27(14.8 \%)$ & $1 / 8(12.5 \%)$ & $14(18.9 \%)$ \\
\hline
\end{tabular}

${ }^{*} P<0.05$.

present in 42 hepatocellular carcinoma (57\%). Telangiectasias were present in $3(4 \%)$, peliosis was present in $6(8 \%)$, and intratumoral inflammatory foci were present in 15 hepatocellular carcinomas $(20 \%)$.

\section{Immunohistochemistry}

Eighteen hepatocellular carcinomas (24\%) had complete lack of liver fatty acid-binding protein expression, 4 of which had steatosis within the 
tumor (Table 3). The 18 liver fatty acid-binding protein-negative cases were more common in men (12:6), negative for alcoholic liver disease or viral hepatitis, and $54 \%$ of the subjects were overweight or obese. These tumors also tended to be large (range: 1-24 cm, mean $9.8 \mathrm{~cm}$ ); 44\% were multifocal, compared with $42 \%$ of the remaining hepatocellular carcinomas that expressed liver fatty acid-binding

Table 2 Pathology of noncirrhotic hepatocellular carcinoma

\begin{tabular}{lc}
\hline Size (cm) & $\begin{array}{c}\text { Mean: } 8.10 \mathrm{~cm} \\
\text { (range: } 1.3-24 \mathrm{~cm})\end{array}$ \\
\hline Multifocalilty & $20(27.0 \%)$ \\
Grade (Edmonson-Steiner) & \\
I & $10(13.5 \%)$ \\
II & $35(47.3 \%)$ \\
III & $17(23.0 \%)$ \\
IV & $12(16.2 \%)$ \\
Histologic subtype & \\
Fibrolamellar & \\
Inflammatory & $2(2.7 \%)$ \\
Scirrhous & $5(6.8 \%)$ \\
Necrosis & $1(1.4 \%)$ \\
Encapsulation & \\
Lymph-vascular invasion & $42(56.8 \%)$ \\
Significant steatosis (tumoral) & $37(50.0 \%)$ \\
Significant steatosis (nontumor & $25(33.8 \%)$ \\
liver) & $7(9.5 \%)$ \\
Telangiectasia & $4(5.4 \%)$ \\
Peliosis & $3(4.1 \%)$ \\
Fibrosis in nontumor liver (stage) & $6(8.1 \%)$ \\
0/1/2/3 & \\
& $20(27.0 \%) / 19$ \\
& $(10.8 \%)$ \\
\hline
\end{tabular}

protein. Among the liver fatty acid-binding proteinnegative tumors, 5 showed bland cytology, a feature common to hepatocellular adenoma. On the other hand, noncirrhotic hepatocellular carcinoma with diffuse liver fatty acid-binding protein immunoreactivity were more often associated with lower histology grades (Edmondson-Steiner grades I and II; $P<0.05)$. Representative staining patterns are shown in Figure 2.

Beta-catenin nuclear and cytoplasmic expression was found in $5 \%$ of cases, and the glutamine synthetase (glutamine synthetase) expression was diffusely and strongly positive in these same cases. The positive cases were all men, aged $62-78$ years (median 64.5 years, mean 67 years), all solitary lesions, with mean tumor size of $8.25 \mathrm{~cm}$ (range, $3.5-14 \mathrm{~cm}$ ). Although advanced age and male gender of the involved subjects make pre-existing hepatocellular adenoma unlikely, it is challenging to completely exclude the possibility. Representative expression patterns are in Figure 3.

In our cohort, 39\% (29 noncirrhotic hepatocellular carcinoma) showed no serum amyloid A immunohistochemistry expression whereas of the $61 \%$ that did, serum amyloid A was focally present in 31 and diffuse in 14. The tumors in the diffusely serum amyloid A-positive group were larger than in the serum amyloid A-negative group (Table 4; $P<0.05$ ). The subjects with diffuse reactivity were younger and were more often overweight or obese; $39 \%$ had diabetes, and up to $36 \%$ had steatosis in the background liver $(P<0.05$ compared with non-serum amyloid A-expressing hepatocellular carcinoma). None of the subjects had viral hepatitis or alcoholic liver disease. The ratio of men to women remained high in both the positive (10:4) and negative (18:11)

Table 3 Liver fatty acid binding protein immunohistochemistry

\begin{tabular}{|c|c|c|c|}
\hline Staining pattern & None $(<1 \%)$ & Focal/patchy $(<80 \%)$ & Diffuse $(\geqslant 80 \%)$ \\
\hline $\mathrm{N}$ & $18(24.3 \%)$ & $24(32.4 \%)$ & $32(43.2 \%)$ \\
\hline Age (year) & 18-80 (median 67.5) & 38-79 (median 65) & 26-83 (median 66) \\
\hline Male: female & $12: 6$ & $17: 7$ & $15: 17$ \\
\hline Viral hepatitis or alcoholic liver disease & 0 & 4 & 1 \\
\hline Diabetes mellitus & $2 / 13(15.4 \%)$ & 7/19 (36.8\%) & $7 / 29(24.1 \%)$ \\
\hline Body mass index (available n) & 13 & 17 & 24 \\
\hline$<25$ & $6(46.2 \%)$ & $3(17.6 \%)$ & $10(41.7 \%)$ \\
\hline $25-29$ & $5(38.5 \%)$ & $6(35.3 \%)$ & $7(29.2 \%)$ \\
\hline$\geqslant 30$ & $2(15.4 \%)$ & $8(47.1 \%)$ & $7(29.2 \%)$ \\
\hline Metabolic syndrome & $5 / 6(83.3 \%)$ & $3 / 7(42.3 \%)$ & $7 / 13(53.8 \%)$ \\
\hline Size $(\mathrm{cm})$ & $1.3-24$ (mean 9.8) & $2.2-15$ (mean 8.0) & $1.5-17$ (mean 7.2) \\
\hline Multifocality & $8(44.4 \%)$ & $4(16.7 \%)$ & $8(25.0 \%)$ \\
\hline Tumor grade* & I: $2(11.1 \%)$ & I: $2(8.3 \%)$ & I: $6(18.8 \%)$ \\
\hline$(P<0.05$ when comparing grades & II:7 (38.9\%), & II: 9 (37.5\%), & II: 19 (59.4\%), \\
\hline \multirow[t]{2}{*}{$\mathrm{I}+\mathrm{II}$ and III + IV) } & III: $5(27.8 \%)$, & III: $8(33.3 \%)$, & III: $4(12.5 \%)$, \\
\hline & IV: $4(22.2 \%)$ & IV: $5(20.8 \%)$ & IV: $3(9.4 \%)$ \\
\hline Steatosis (tumor) & $2(11.1 \%)$ & $2(8.3 \%)$ & $8(25.0 \%)$ \\
\hline Steatosis (nontumor liver) & $3(16.7 \%)$ & $6(25.0 \%)$ & $6(18.8 \%)$ \\
\hline \multirow[t]{4}{*}{ Fibrosis } & $0: 5(27.8 \%)$ & $0: 5(20.8 \%)$ & $0: 10(31.3 \%)$ \\
\hline & 1: $4(22.2 \%)$ & 1: $8(33.3 \%)$ & 1: $7(21.9 \%)$ \\
\hline & $2: 8(44.4 \%)$ & 2: $6(25.0 \%)$ & $2: 13(40.6 \%)$ \\
\hline & 3: $1(5.6 \%)$ & $3: 5(20.8 \%)$ & $3: 2(6.3 \%)$ \\
\hline
\end{tabular}

${ }^{*} P<0.05$. 
a
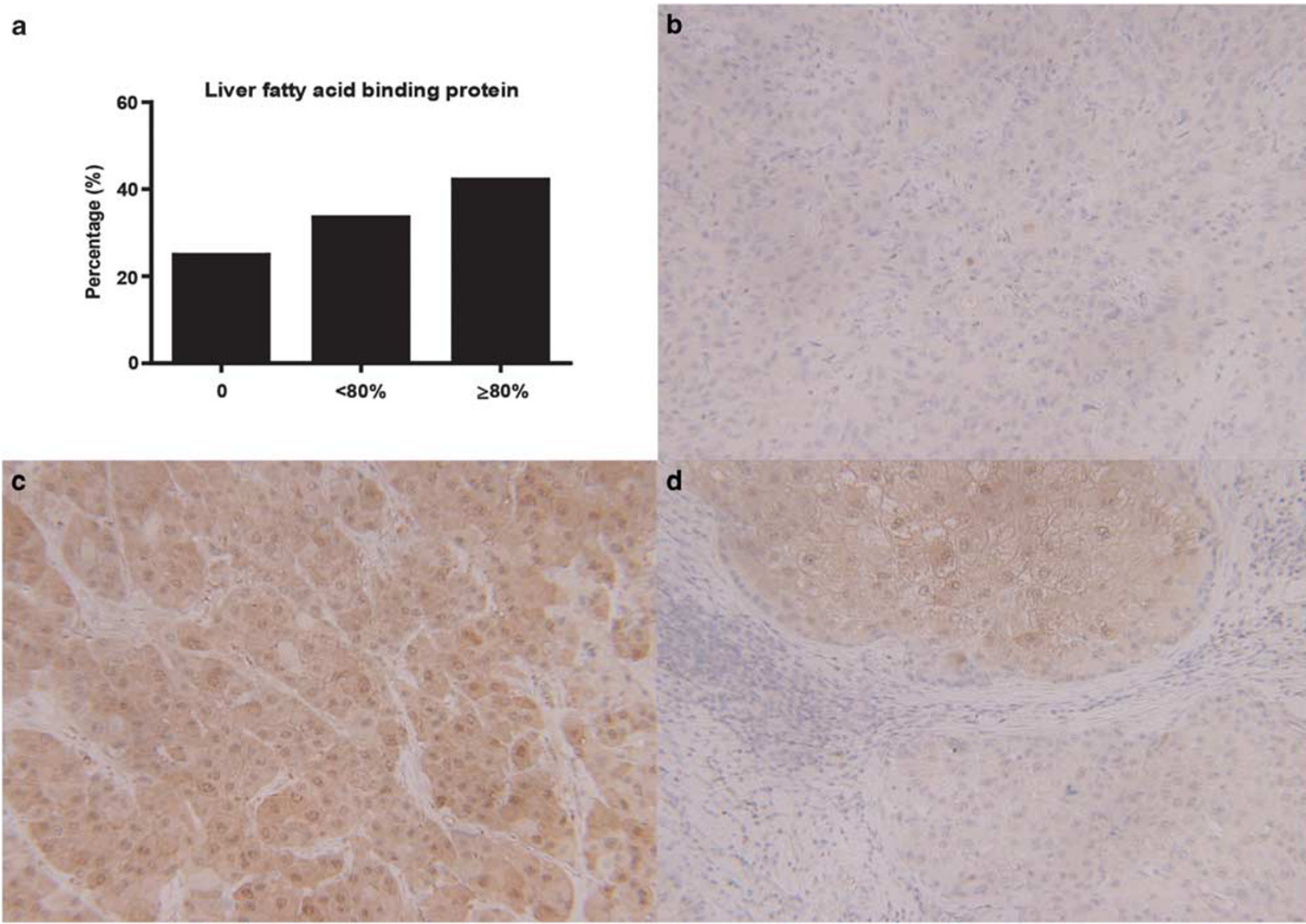

Figure 2 Staining patterns of liver fatty acid-binding protein. (a) Bar graph; (b-d) representative photomicrographs of different staining patterns, including (b) complete lack of expression; (c) no lack of expression; and (d) focal expression.

tumors. The serum amyloid A-positive hepatocellular carcinomas tended to be lower EdmondsonSteiner grade (I, II). Unlike reports of serum amyloid A-positive hepatocellular adenoma, 21\% of serum amyloid A-positive hepatocellular carcinomas also had tumoral steatosis. Interestingly, 4 of the 14 diffusely serum amyloid A-positive hepatocellular carcinomas showed morphologic features often associated with hepatocellular adenoma, including various inflammation, telangiectasias and peliosis. This includes a 30-year-old woman and three men aged 56, 58, and 75 years old, respectively. Representative staining patterns are shown in Figure 4.

In uninvolved liver, glutamine synthetase immunohistochemistry reflects nitrogen metabolism and is thus typically present only in the immediate zone 3 rim (perivenular) hepatocytes. In hepatocellular adenoma, nonzonal (diffuse or patchy) glutamine synthetase stain pattern with loss of zonation may occur, whereas diffuse and intense reactivity is highly suggestive of hepatocellular carcinoma in the appropriate clinical setting. ${ }^{23}$ In the current study, the majority of hepatocellular carcinoma (50 cases; $68 \%$ ) showed diffuse reactivity within the tumor, whereas 5 cases showed patchy reactivity (7\%)
(Table 5). Eighteen cases (24\%) demonstrated a less regular, more heterogeneous but intense pattern, but none were 'map-like' as seen with focal nodular hyperplasia. Only 1 case had no expression of glutamine synthetase $(1 \%)$. The patient was an 18-year-old man with serum amyloid A-positive, $\beta$-catenin-negative hepatocellular carcinoma, who had no underlying risk factors for hepatocellular carcinoma or adenoma. Interestingly, the 5 cases with patchy glutamine synthetase staining pattern did not have fibrosis $(P<0.05)$, whereas the 8 livers with background bridging fibrosis had more irregular reactivity $(P<0.05)$. Patients whose tumors showed heterogeneous staining patterns were more commonly overweight or obese. Representative staining patterns are shown in Figure 5.

The expression of glypican-3, a fetal oncoprotein that has gained interest in distinguishing hepatocellular adenoma from carcinoma when positive, ${ }^{24-26}$ was evaluated. The majority of the cases $(70 \%)$ showed varying extents and degrees of glypican-3 expression, but 22 tumors (30\%) were negative. The reactivity patterns in glypican-3-expressing tumors were quite variable: patchy in $30 \%$, diffuse in $38 \%$, and heterogeneous in $3 \%$. Most of the glypican3-negative hepatocellular carcinomas were grade I 
a
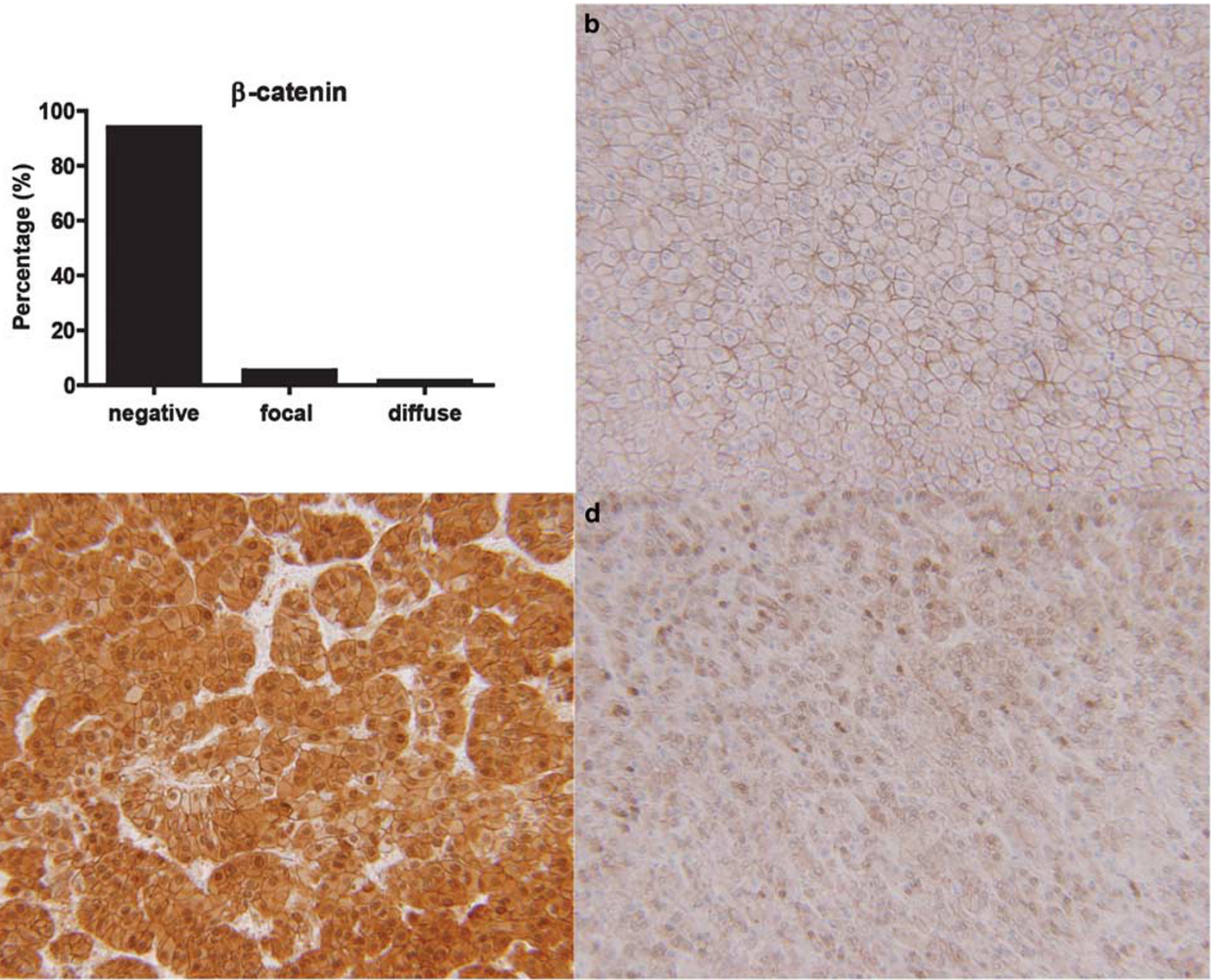

Figure 3 Staining patterns of $\beta$-catenin. (a) Bar graph; (b-d) representative photomicrographs of different $\beta$-catenin staining patterns, including (b) membranous staining indicating inactivated $\beta$-catenin; (c) nuclear and cytoplasmic staining indicating activated $\beta$-catenin; (d) heterogeneous staining patterns with areas of nuclear staining admixed with membranous staining.

$(23 \%)$ or II $(50 \%)$. No correlation with tumor size and glypican-3 expression was noted, but all eight cases with stage 3 fibrosis were positive for glypican-3 reactivity $(P<0.05)$. Glypican-3 was negative in serum amyloid A-positive tumors, and positive in serum amyloid A-negative tumors $(P<0.05)$. Glypican-3-positive noncirrhotic hepatocellular carcinomas were also more commonly found to have strong nuclear p53 immunohistochemistry $(P<0.05)$.

Overall, it was concluded that 22 noncirrhotic hepatocellular carcinoma $(29.7 \%)$ were potentially derived from hepatocellular adenoma based on the clinical, histologic features, and immunoprofile, including the following: (1) morphologic suggestion of co-existing noncirrhotic hepatocellular carcinoma and adenoma in the same lesion $(n=10)$; (2) steatotic tumor lacking liver fatty acid-binding protein staining $(n=4)$; (3) diffuse serum amyloid A expression in noncirrhotic hepatocellular carcinoma that showed morphologic features of inflammatory adenoma within the tumor $(n=4)$; (4) nuclear and/or cytoplasmic beta-catenin with diffuse glutamine synthetase staining in the tumor $(n=4)$.

Mutation of p53 tumor suppressor gene has been implicated in HCC carcinogenesis. ${ }^{27}$ Overall, 30\% of the noncirrhotic hepatocellular carcinoma in our series showed certain degrees of nuclear p53 labeling. Ten cases showed p53 immunoreactivity in $\geqslant 10 \%$ of tumor nuclei; the remainder were negative $(58 \%)$, or had very low expression $(<1 \%$ of cells), or expression in 1-10\% of tumor nuclei (12 cases). Other than with the glypican-3 and K19 stains, as described below, p53 immunohistochemistry had no correlation with the number of tumor, tumor size, or expression of other immunohistochemistry markers.

K19 expression has been proposed as a poor prognostic indicator in cirrhotic hepatocellular carcinoma. ${ }^{28-30}$ Using $5 \%$ positivity as a cutoff, $88 \%$ of noncirrhotic hepatocellular carcinoma were negative. Of the 9 hepatocellular carcinoma with K19 positivity in $\geqslant 5 \%$, 4 were $<20 \%$; 5 showed 
Table 4 Serum amyloid A immunohistochemistry

\begin{tabular}{|c|c|c|c|}
\hline Staining pattern & None $(0 \%)$ & Focal/patchy $(<80 \%)$ & Diffuse $(\geqslant 80 \%)$ \\
\hline $\mathrm{N}$ & $29(39.2 \%)$ & $31(41.9 \%)$ & $14(18.9 \%)$ \\
\hline Age (year) & 26-83 (median 69) & 32-80 (median 65) & 18-75 (median 59.5) \\
\hline Male: female & 18: 11 & 16: 15 & 10: 4 \\
\hline Viral hepatitis or alcoholic liver disease & 3 & 2 & 0 \\
\hline Diabetes mellitus & $5 / 22(22.7 \%)$ & $6 / 30(20.0 \%)$ & $5 / 13(38.5 \%)$ \\
\hline Body mass index (available $n$ ) & 19 & 23 & 12 \\
\hline$<25$ & $7(36.8 \%)$ & $10(43.5 \%)$ & $2(16.7 \%)$ \\
\hline $25-29$ & $6(31.6 \%)$ & $7(30.4 \%)$ & $5(41.7 \%)$ \\
\hline$\geqslant 30$ & $6(31.6 \%)$ & $6(26.1 \%)$ & $5(41.7 \%)$ \\
\hline Metabolic syndrome & $4 / 4(100.0 \%)$ & $6 / 13(46.2 \%)$ & $5 / 9(55.6 \%)$ \\
\hline Size $(\mathrm{cm})^{*}$ & $1.3-19($ mean 7.9$)$ & $1.5-13.5$ (mean 6.8) & $2.2-24$ (mean 11.4) \\
\hline Multifocality & $8(27.6 \%)$ & $9(29.0 \%)$ & $3(21.4 \%)$ \\
\hline \multirow[t]{4}{*}{ Tumor grade } & I: $1(3.4 \%)$, & I: $5(16.1 \%)$ & I: $4(28.6 \%)$ \\
\hline & II: $15(51.7 \%)$, & II: 13 (41.9\%), & II: 7 (50.0\%), \\
\hline & III: $7(24.1 \%)$, & III: $9(29.0 \%)$, & III: $1(7.1 \%)$ \\
\hline & IV: $6(20.7 \%)$ & IV: $4(13.8 \%)$ & IV: $2(14.3 \%)$ \\
\hline Steatosis (tumor) & $4(13.8 \%)$ & $5(16.1 \%)$ & $3(21.4 \%)$ \\
\hline Steatosis (liver)* & $2(6.9 \%)$ & $8(25.8 \%)$ & $5(35.7 \%)$ \\
\hline \multirow[t]{4}{*}{ Fibrosis } & $0: 10(34.5 \%)$ & $0: 7(22.6 \%)$ & $0: 4(28.6 \%)$ \\
\hline & 1: $8(27.6 \%)$ & 1: $5(16.1 \%)$ & 1: $5(35.7 \%)$ \\
\hline & $2: 7(24.1 \%)$ & 2: $16(51.6 \%)$ & $2: 4(28.6 \%)$ \\
\hline & $3: 4(13.8 \%)$ & $3: 3(9.7 \%)$ & $3: 1(7.1 \%)$ \\
\hline
\end{tabular}

${ }^{*} P<0.05$.

$\mathrm{K} 19 \geqslant 20 \%(20 \%$ in $1,30 \%$ in $1,90-95 \%$ in 3$)$. Expression of K19 $\geqslant 5 \%$ correlated with less tumor encapsulation and with the presence of p53 mutation (both $P<0.05$ ), but not with tumor grade, lymph-vascular space invasion, tumor size, multifocality, any other tested markers, recurrence-free survival or overall survival. The 5 cases with K19 $\geqslant 20 \%$ tended to have worse prognosis but that did not reach statistical significance. Therefore, in this group of noncirrhotic hepatocellular carcinoma, K19 expression is not considered a prognostic marker.

When data were stratified by stage of fibrosis (Table 1), there were two main findings. The tumors in the setting of no or minimal fibrosis (stage 0-1) showed greater tumor size than those in mild or moderate fibrotic livers (stage $2-3)(P<0.05)$. Similarly, none of the tumors in stage 0-1 group showed moderate or marked intratumoral steatosis, whereas 7 of 35 tumors in the stage $2-3$ group did $(P<0.05)$.

Previously reported prognosis of resected noncirrhotic hepatocellular carcinoma is up to 31 months of recurrence-free survival and 3 years of overall survival (reviewed in the study by Laurent et $a l^{18}$ ). The noncirrhotic hepatocellular carcinoma patients in the current study had a median recurrence-free survival of 32.78 months and an overall survival of 38.83 months (Figures 6a and b). Our data are consistent with the reported prognosis of noncirrhotic hepatocellular carcinoma patients treated by resection. ${ }^{18,31}$

\section{Discussion}

Hepatocellular carcinoma in noncirrhotic liver is uncommon, yet may pose diagnostic challenges and conceptual challenges as to etiopathogenesis. The literature has documented that $3-6 \%$ of resected hepatocellular adenomas show areas of hepatocellular carcinoma, and that tumor size $>5 \mathrm{~cm}$ and male gender are risks, ${ }^{32,33}$ but it is not known if noncirrhotic hepatocellular carcinomas in general are the result of hepatocellular adenoma transformation or represent de novo carcinoma. Farges et.al ${ }^{34}$ showed malignant transformation in $10.5 \%$ of 218 hepatocellular adenoma studied over a recent 10-year period. The calculated risk of malignant transformation of $4 \%$ in women had remained stable, but the $47 \%$ risk in men increased along with the prevalence of metabolic syndrome. In a smaller series, 3 of the 17 resected hepatocellular adenoma with malignant transformation were all in reproductive-aged women, as is common for hepatocellular adenoma; one hepatocellular carcinoma and none of the hepatocellular adenoma had evidence of mutated p53. ${ }^{35}$

In this study, we characterized the etiology, morphology, immunohistochemistry, and survival in 72 patients with 74 noncirrhotic hepatocellular carcinoma treated by resection spanning 20 years in our institution. Several points are worth noting. Although over half (59\%) of the patients were men, average age 64 years, just as in cirrhotic hepatocellular carcinoma, women predominated by 8:1 in the $<50$-year-old-age group. This is a unique and important finding, as hepatocellular adenoma also occur predominantly in women, and in a younger age group. ${ }^{10}$ This finding in our series may lend insight into pathogenesis; from a diagnostic perspective, on the other hand, it also suggests that female gender is not always reliable in favoring hepatocellular adenoma over noncirrhotic 
a
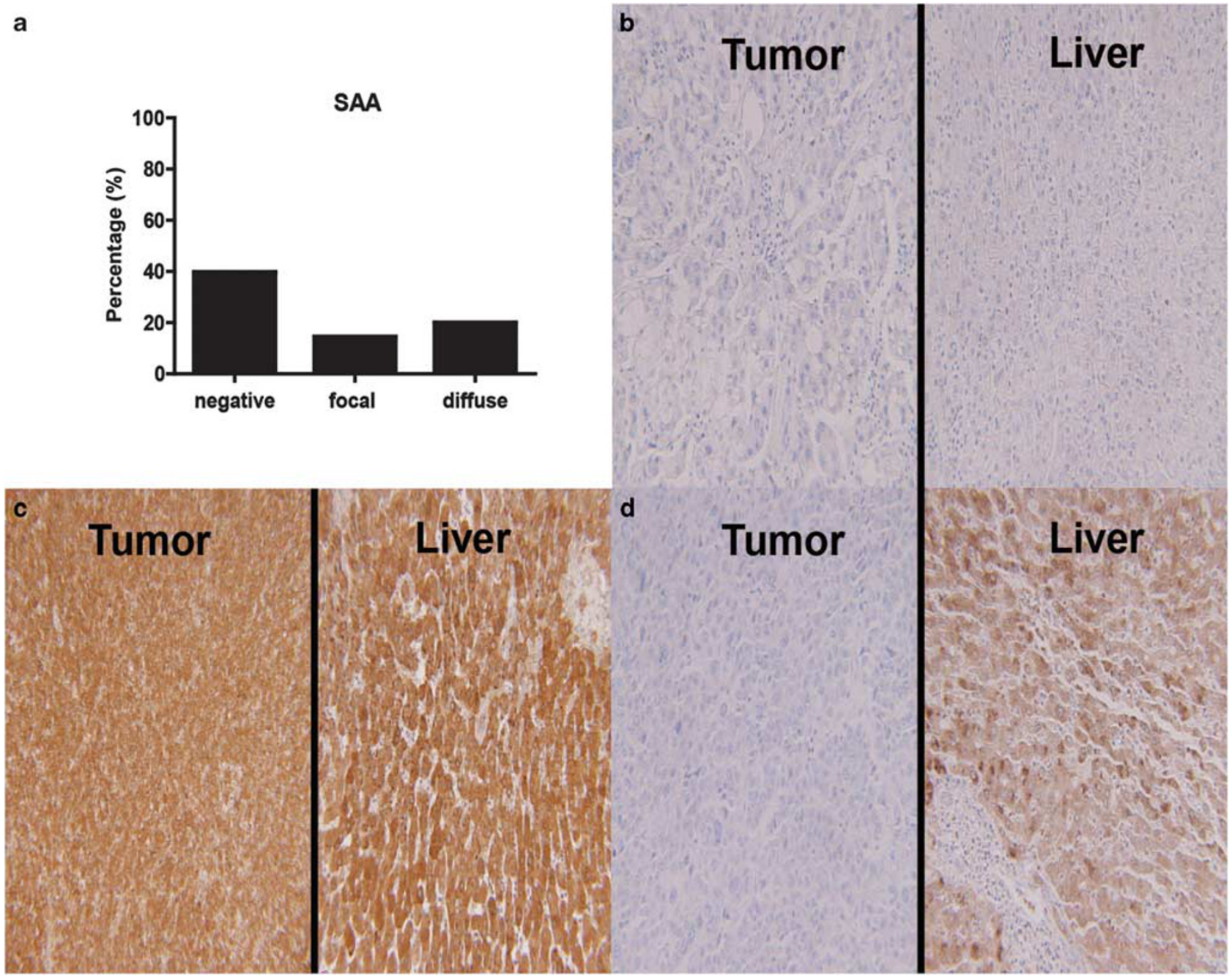

Tumor

Figure 4 Staining patterns of serum amyloid A. (a) Bar graph; (b-d) representative photomicrographs of different serum amyloid A staining patterns, including (b) complete lack of expression in both hepatocellular carcinoma and uninvolved liver; (c) expression in both hepatocellular carcinoma and uninvolved liver; (d) lack of expression in hepatocellular carcinoma but no lack of expression in uninvolved liver.

hepatocellular carcinoma in the >50-year-old age group. In contrast, hepatocellular adenoma is rare in men, and the presence of a large liver mass in noncirrhotic liver is more likely to be hepatocellular carcinoma, especially in the >50-year-old age man.

Two-thirds of the patients were overweight or obese, $25 \%$ had diabetes, and $58 \%$ had metabolic syndrome. While nonalcoholic fatty liver disease was common, the amount of steatosis was rarely higher than grade 1 and steatohepatitis in the nontumor liver was not seen in any case. A recent French study recognized the strong association of hepatocellular carcinoma with metabolic syndrome in noncirrhotic livers. ${ }^{3}$ Other studies have also concluded that obesity and diabetes are associated with hepatocellular carcinoma. ${ }^{36}$ In our patient cohort, two-thirds were overweight or obese, which likely reflects the high prevalence of obesity and overweight in the US population, ${ }^{37}$ higher than in France. ${ }^{38}$ Thus it is still unclear whether it is the prevalence of obesity per se, a recognized systemic chronic pro-inflammatory condition, that predisposes to hepatocellular carcinoma, or if obesity is a co-factor in initiation and progression. Obesity and metabolic syndrome are both associated with hyperinsulinemia, a pro-inflammatory and proliferative state that has been linked to hepatocellular carcinoma. ${ }^{39,40}$

The majority of the patients (93\%) had no evidence of viral hepatitis or alcoholic liver disease. The majority of the background livers showed periportal (stage 2) or less fibrosis. No significant differences beyond greater tumor size in livers with lower stages of fibrosis, and more tumoral steatosis in hepatocellular carcinoma with higher background fibrosis were noted between background livers in this cohort.

The potential expression of recently proposed immunohistochemistry markers for hepatocellular adenoma, ${ }^{10-13}$ other than $\beta$-catenin and glutamine synthetase, in noncirrhotic hepatocellular carcinoma is relatively unknown; ours is the first 
Table 5 Glutamine synthetase immunohistochemistry

\begin{tabular}{|c|c|c|c|c|}
\hline Staining pattern & Heterogeneous & Focal/patchy & Diffuse & Negative \\
\hline $\mathrm{N}$ & $18(24.3 \%)$ & $5(6.8 \%)$ & $50(67.6 \%)$ & $1(1.4 \%)$ \\
\hline Age (year) & 32-83 (median 70.5) & 38-82 (median 65) & 26-80 (median 65.5) & 18 \\
\hline Male: female & 10: 8 & 3: 2 & 30: 20 & M \\
\hline Viral hepatitis or alcoholic liver disease & 2 & 0 & 4 & 0 \\
\hline Diabetes mellitus & $4 / 15(26.7)$ & $2 / 6(33.3 \%)$ & $10 / 45(22.2 \%)$ & 0 \\
\hline Body mass index (available $n$ ) & 10 & 5 & 38 & 1 \\
\hline$<25$ & $3(30.0 \%)$ & $2(40.0 \%)$ & $13(34.2 \%)$ & 1 \\
\hline $25-29$ & $4(40.0 \%)$ & $1(20.0 \%)$ & $13(34.2 \%)$ & 0 \\
\hline$\geqslant 30$ & $3(30.0 \%)$ & $2(40.0 \%)$ & $12(31.6 \%)$ & 0 \\
\hline Metabolic syndrome & $3 / 6(50.0 \%)$ & $1 / 2(50.0 \%)$ & $11 / 18(61.1 \%)$ & N/A \\
\hline Size $(\mathrm{cm})$ & $2.5-15$ (mean 7.9) & 4.5-17 (mean 8.0) & $1.3-24($ mean 8.1) & 11.5 \\
\hline Multifocality & $6(33.3 \%)$ & 0 & $14(28.0 \%)$ & 0 \\
\hline \multirow[t]{4}{*}{ Tumor grade } & $\mathrm{I}: 3(16.7 \%)$ & I:0, & $\mathrm{I}: 7(14.0 \%)$ & \multirow[t]{4}{*}{ III } \\
\hline & II:10 (55.6\%), & II:4 (80.0\%), & II:21 $(42.0 \%)$ & \\
\hline & III:2 (11.1\%), & III:0, & III:14 $(28.0 \%)$, & \\
\hline & IV:3 (16.7\%) & IV:1 (20.0\%) & IV:8 $(16.0 \%)$ & \\
\hline Steatosis (tumor) & $4(22.2 \%)$ & 0 & $8(16.0 \%)$ & 0 \\
\hline Steatosis (liver) & $3(16.7 \%)$ & $1(20.0 \%)$ & $11(22.0 \%)$ & 0 \\
\hline \multirow{4}{*}{ Fibrosis* } & $0: 2(11.1 \%), *$ & $0: 5(100.0 \%), *$ & $0: 13(26.0 \%),{ }^{*}$ & \multirow[t]{4}{*}{2} \\
\hline & $1: 5(27.8 \%)$ & $1: 0$ & $1: 14(28.0 \%)$ & \\
\hline & $2: 8(44.4 \%)$ & $2: 0$ & $2: 18(36.0 \%)$ & \\
\hline & $3: 3(16.7 \%)$ & $3: 0$ & $3: 5(10.0 \%)$ & \\
\hline
\end{tabular}

${ }^{*} P<0.05$.

study to systematically analyze them in this setting. The study was conducted under the assumptions that (1) noncirrhotic and cirrhotic hepatocellular carcinoma have distinct mechanistic pathogenic pathways, and (2) if the hepatocellular carcinoma had developed from malignant transformation of hepatocellular adenoma, the immunohistochemistry markers of the hepatocellular adenoma could possibly be retained. No a priori assumptions were made as to gender or age for the initial lesion (hepatocellular adenoma or carcinoma), as the lesions being evaluated represent the final stage of carcinogenesis. The current study demonstrated expression (or loss of) of markers in up to $80 \%$ of the noncirrhotic hepatocellular carcinomas: $24 \%$ showed loss of liver fatty acid binding protein, and $>50 \%$ showed some degree of serum amyloid A expression. Five percent had nuclear and cytoplasmic expression of $\beta$-catenin, and all of the latter also had diffuse glutamine synthetase positivity. In fact, all but 1 noncirrhotic hepatocellular carcinoma expressed glutamine synthetase. Only $50 \%$ of the noncirrhotic hepatocellular carcinoma were encapsulated, as with hepatocellular adenoma, and $39 \%$ had other morphologic features found in hepatocellular adenoma such as peliosis, inflammatory foci, and bland cytology. However, when all morphologic and immunophenotypic features were considered, only $29.7 \%$ of the noncirrhotic hepatocellular carcinomas could be potentially classified as malignant transformation of an hepatocellular adenoma.

While it has been shown that loss of detectable liver fatty acid-binding protein expression by tumoral hepatocytes by immunohistochemistry correlates with bi-allelic hepatocyte nuclear factor$1 \alpha$ mutation, ${ }^{11}$ it is not known if this is the case in hepatocellular carcinoma. Similar to hepatocyte nuclear factor-1 $\alpha$-mutated hepatocellular adenoma, the liver fatty acid-binding protein-negative noncirrhotic hepatocellular carcinomas were slightly more often multiple. Nonetheless, among the $24 \%$ of noncirrhotic hepatocellular carcinoma that showed complete loss of liver fatty acid-binding protein, only 4 cases contained intratumoral steatosis. Serum amyloid A, an acute phase reactant expressed by hepatocytes, is a marker for inflammatory hepatocellular adenoma and showed at least weak expression in $60 \%$ of noncirrhotic hepatocellular carcinoma. Among the serum amyloid A-expressing HCCs, 33\% showed morphologic suggestions of hepatocellular adenoma or had various degrees of intratumoral inflammation; serum amyloid A positivity correlated with tumor size.

Other markers of interest in hepatocellular carcinoma in our study included glypican-3, p53 and K19. The first was more common in high than lowgrade tumors, and positivity correlated with intratumoral p53 expression. The inverse correlation of glypican-3 and serum amyloid A expression suggests that serum amyloid A-expressing noncirrhotic hepatocellular carcinomas may have different carcinogenic pathways. The majority of the noncirrhotic hepatocellular carcinomas did not show evidence of p53 mutation or $\geqslant 5 \%$ K19 expression; thus, neither was considered to be prognostic.

The overall 5-year survival for resected noncirrhotic hepatocellular carcinoma is reported to be $30-50 \% .{ }^{18,31}$ Our data are consistent with these published results. Lack of early detection likely has a significant role in this poor overall survival. Surgery, in the form of transplantation or resection, remains the treatment modality of choice in noncirrhotic hepatocellular carcinoma patients. 

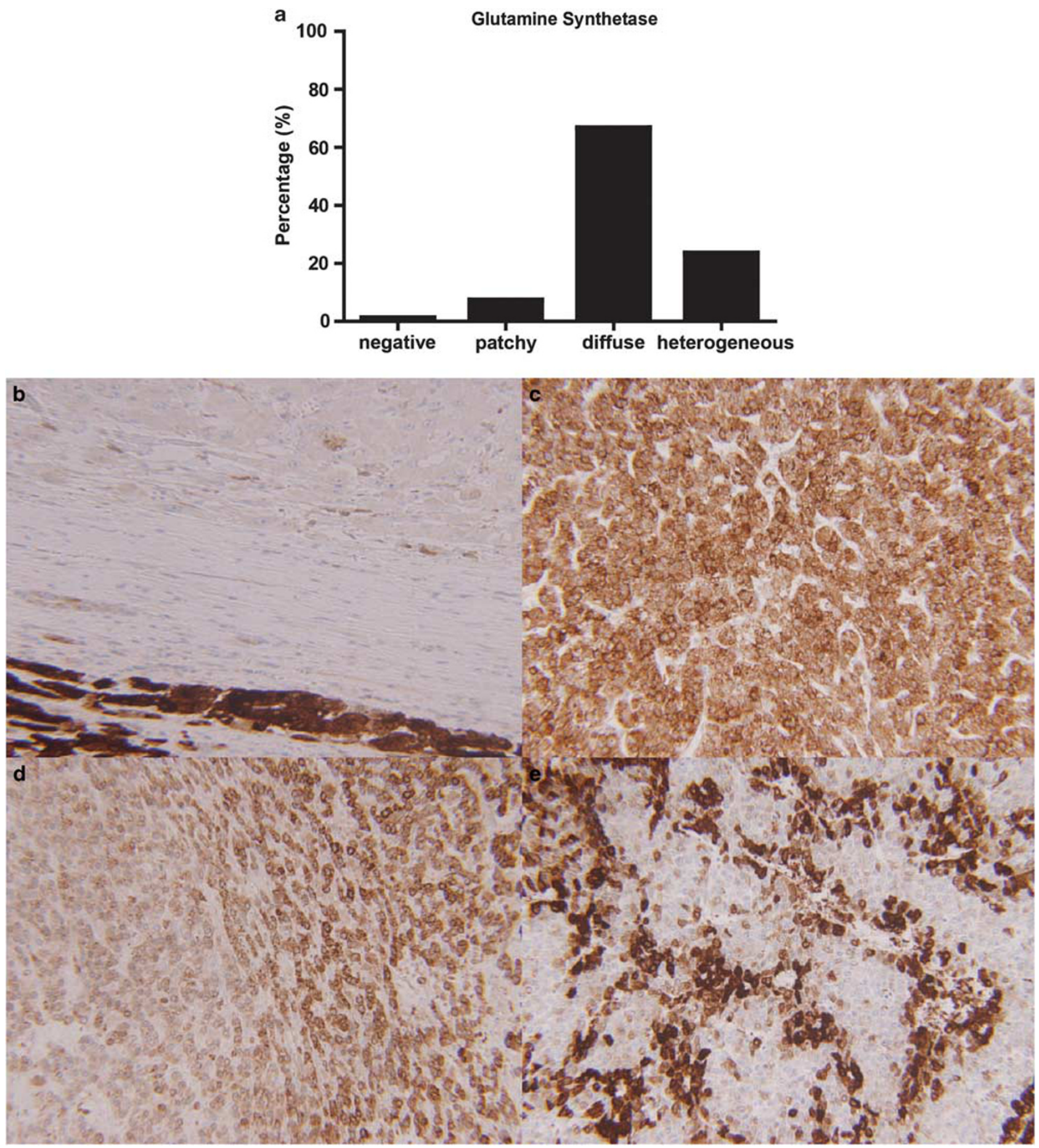

Figure 5 Staining patterns of glutamine synthetase. (a) Bar graph; (b-e) representative photomicrographs of different staining patterns, including (b) complete lack of expression; (c) diffuse, strong expression; (d) focal expression; and (e) heterogeneous expression.

One limitation of this study is that of retrospective analysis of tumor and background liver tissue sampling. Tissue sampling had been performed according to contemporaneous protocols for routine patient care. In addition, complete clinical information for the evaluation for metabolic syndrome is only available for a fraction of the patients and therefore a firm conclusion cannot be drawn; none- theless, our finding of association with metabolic syndrome is consistent with other studies. Another caveat is that the expression (or lack of) certain markers may be due to etiologies other than adenoma transformation. However, as no preclinical model is currently available to address the etiopathogenesis of noncirrhotic hepatocellular carcinoma, these immunohistochemistry markers, 

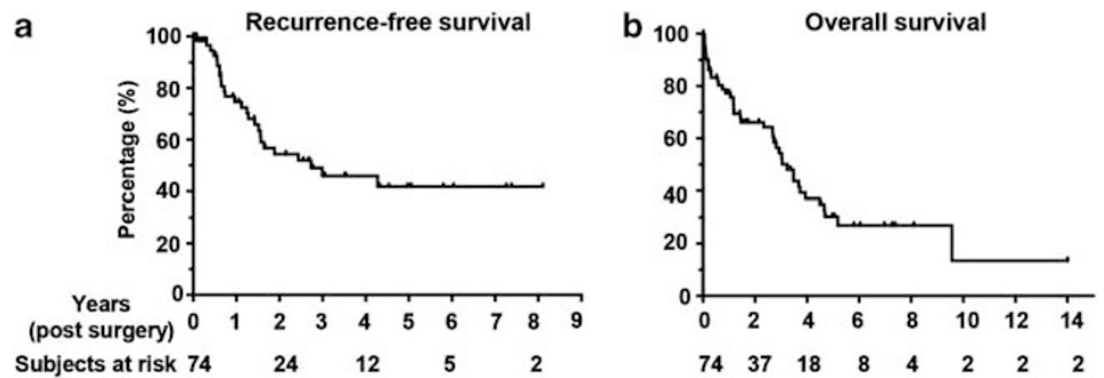

Figure 6 (a) Recurrence-free survival and (b) overall survival of patients with noncirrhotic hepatocellular carcinoma.

together with clinical and morphological correlates, are the best available tools to attempt to address possible relationships between hepatocellular adenoma and noncirrhotic hepatocellular carcinoma to date. Thus, the importance of interpreting the immunoprofile in conjunction with clinical and other histologic features cannot be overemphasized. Future studies involving sequencing (eg, for hepatocyte nuclear factor- $1 \alpha$ mutation in noncirrhotic hepatocellular carcinoma with loss of liver fatty acid binding protein expression) may provide more insight. Analyzing additional cases will allow us to perform multivariate analysis. Finally, we recognize this approach serves as one of many approaches to evaluate the possibility of transformation of hepatocellular adenoma to carcinoma in noncirrhotic livers.

In summary, we present a clinical-pathological study in a large noncirrhotic hepatocellular carcinoma population. We utilized detailed characterization of immunohistochemistry markers currently used to subclassify hepatocellular adenoma in conjunction with morphology to detect possible derivation or association with prior hepatocellular adenoma. Our data suggest that the majority of noncirrhotic hepatocellular carcinoma in our series were de novo and did not represent malignant transformation from hepatocellular adenoma. A unique observation was the predominance of women in the <50-year-old group of noncirrhotic hepatocellular carcinoma, and we confirmed an association of metabolic syndrome with noncirrhotic hepatocellular carcinoma in men. Future directions include identification of contributing factors and genetic and molecular markers, keeping the potential gender and age-related differences in mind. Longitudinal studies with regular screening and follow-up in patients with metabolic syndrome will also provide more insight into the potential relationship between hepatocellular adenoma and noncirrhotic hepatocellular carcinoma.

\section{Acknowledgements}

This work was supported, in part, by an American Medical Association Foundation Seed Grant (TCL). We wish to thank Dr Neha Dahiya and Jianping Li for technical support.

\section{Disclosure/conflict of interest}

The authors declare no conflict of interest.

\section{References}

1 Bruix J, Llovet JM. Two decades of advances in hepatocellular carcinoma research. Semin Liver Dis 2010;30:1-2.

2 Ascha MS, Hanouneh IA, Lopez R, et al. The incidence and risk factors of hepatocellular carcinoma in patients with nonalcoholic steatohepatitis. Hepatology 2010;51: 1972-1978.

3 Paradis V, Zalinski S, Chelbi E, et al. Hepatocellular carcinomas in patients with metabolic syndrome often develop without significant liver fibrosis: a pathological analysis. Hepatology 2009;49:851-859.

4 Sanyal A, Poklepovic A, Moyneur E, et al. Populationbased risk factors and resource utilization for HCC: US perspective. Curr Med Res Opin 2010;26:2183-2191.

5 Llovet JM, Burroughs A, Bruix J. Hepatocellular carcinoma. Lancet 2003;362:1907-1917.

6 Brancatelli G, Federle MP, Grazioli L, et al. Hepatocellular carcinoma in noncirrhotic liver: CT, clinical, and pathologic findings in 39 U.S. residents. Radiology 2002;222:89-94.

7 Trevisani F, Frigerio M, Santi V, et al. Hepatocellular carcinoma in non-cirrhotic liver: a reappraisal. Dig Liver Dis 2010;42:341-347.

8 Bruix J, Sherman M. Management of hepatocellular carcinoma: an update. Hepatology 2011;53:1020-1022.

9 Sherman M. Hepatocellular carcinoma: screening and staging. Clin Liver Dis 2011;15:323-334.

10 Bioulac-Sage P, Balabaud C, Zucman-Rossi J. Subtype classification of hepatocellular adenoma. Dig Surg 2010;27:39-45.

11 Zucman-Rossi J, Jeannot E, Nhieu JT, et al. Genotypephenotype correlation in hepatocellular adenoma: new classification and relationship with HCC. Hepatology 2006;43:515-524.

12 Bioulac-Sage P, Cubel G, Balabaud C, et al. Revisiting the pathology of resected benign hepatocellular nodules using new immunohistochemical markers. Semin Liver Dis 2011;31:91-103.

13 Bioulac-Sage P, Rebouissou S, Thomas C, et al. Hepatocellular adenoma subtype classification using molecular markers and immunohistochemistry. Hepatology 2007;46:740-748.

14 Evason KJ, Grenert JP, Ferrell LD, et al. Atypical hepatocellular adenoma-like neoplasms with betacatenin activation show cytogenetic alterations similar 
to well-differentiated hepatocellular carcinomas. Hum Pathol 2013;44:750-758.

15 Paradis V, Benzekri A, Dargere D, et al. Telangiectatic focal nodular hyperplasia: a variant of hepatocellular adenoma. Gastroenterology 2004;126:1323-1329.

16 Rebouissou S, Amessou M, Couchy G, et al. Frequent in-frame somatic deletions activate gp130 in inflammatory hepatocellular tumours. Nature 2009;457: 200-204.

17 Pilati C, Amessou M, Bihl MP, et al. Somatic mutations activating STAT3 in human inflammatory hepatocellular adenomas. J Exp Med 2011;208:1359-1366.

18 Laurent C, Blanc JF, Nobili S, et al. Prognostic factors and longterm survival after hepatic resection for hepatocellular carcinoma originating from noncirrhotic liver. J Am Coll Surg 2005;201:656-662.

19 Theise ND, Curado MP, Franceschi S, et al. Hepatocellular Carcinoma. IARC: Lyon, 2010.

20 Scheuer PJ. Classification of chronic viral hepatitis: a need for reassessment. J Hepatol 1991;13:372-374.

21 Kleiner DE, Brunt EM, Van Natta M, et al. Design and validation of a histological scoring system for nonalcoholic fatty liver disease. Hepatology 2005;41: 1313-1321.

22 Expert Panel on Detection, Evaluation, and Treatment of High Blood Cholesterol in Adults. Panel. Executive Summary of The Third Report of The National Cholesterol Education Program (NCEP) Expert Panel on Detection, Evaluation, And Treatment of High Blood Cholesterol In Adults (Adult Treatment Panel III). JAMA 2001;285:2486-2497.

23 Di Tommaso L, Franchi G, Park YN, et al. Diagnostic value of HSP70, glypican 3, and glutamine synthetase in hepatocellular nodules in cirrhosis. Hepatology 2007;45:725-734.

24 Yamauchi N, Watanabe A, Hishinuma M, et al. The glypican 3 oncofetal protein is a promising diagnostic marker for hepatocellular carcinoma. Mod Pathol 2005;18:1591-1598.

25 Shafizadeh N, Ferrell LD, Kakar S. Utility and limitations of glypican-3 expression for the diagnosis of hepatocellular carcinoma at both ends of the differentiation spectrum. Mod Pathol 2008;21: 1011-1018.

26 Coston WM, Loera S, Lau SK, et al. Distinction of hepatocellular carcinoma from benign hepatic mimickers using Glypican-3 and CD34 immunohistochemistry. Am J Surg Pathol 2008;32:433-444.
27 Villanueva A, Hoshida Y. Depicting the role of TP53 in hepatocellular carcinoma progression. J Hepatol 2011; 55:724-725.

28 Uenishi T, Kubo S, Yamamoto T, et al. Cytokeratin 19 expression in hepatocellular carcinoma predicts early postoperative recurrence. Cancer Sci 2003;94:851-857.

29 Durnez A, Verslype C, Nevens F, et al. The clinicopathological and prognostic relevance of cytokeratin 7 and 19 expression in hepatocellular carcinoma. A possible progenitor cell origin. Histopathology 2006;49:138-151.

30 Yang XR, Xu Y, Shi GM, et al. Cytokeratin 10 and cytokeratin 19: predictive markers for poor prognosis in hepatocellular carcinoma patients after curative resection. Clin Cancer Res 2008;14:3850-3859.

31 Dupont-Bierre E, Compagnon P, Raoul JL, et al. Resection of hepatocellular carcinoma in noncirrhotic liver: analysis of risk factors for survival. J Am Coll Surg 2005;201:663-670.

32 Stoot JH, Coelen RJ, De Jong MC, et al. Malignant transformation of hepatocellular adenomas into hepatocellular carcinomas: a systematic review including more than 1600 adenoma cases. HPB (Oxford) 2010; 12:509-522.

33 Farges O, Dokmak S. Malignant transformation of liver adenoma: an analysis of the literature. Dig Surg 2010; $27: 32-38$.

34 Farges O, Ferreira N, Dokmak S, et al. Changing trends in malignant transformation of hepatocellular adenoma. Gut 2011;60:85-89.

35 Micchelli ST, Vivekanandan P, Boitnott JK, et al. Malignant transformation of hepatic adenomas. Mod Pathol 2008;21:491-497.

36 Starley BQ, Calcagno CJ, Harrison SA. Nonalcoholic fatty liver disease and hepatocellular carcinoma: a weighty connection. Hepatology 2010;51:1820-1832.

37 National health and nutrition examination survey 20052006. Accessed at http://www.cdc.gov/nchs/products/ pubs/pubd/hestats/overweight/overweight_adult.pdf. 2009.

38 Balkau B, Vernay M, Mhamdi L, et al. The incidence and persistence of the NCEP (National Cholesterol Education Program) metabolic syndrome. The French D.E.S.I.R. study. Diabetes Metab 2003;29:526-532.

39 Siegel AB, Zhu AX. Metabolic syndrome and hepatocellular carcinoma: two growing epidemics with a potential link. Cancer 2009;115:5651-5661.

40 Bugianesi E. Non-alcoholic steatohepatitis and cancer. Clin Liver Dis 2007;11:191-207; (x-xi). 